\title{
Applications to Photochemistry
}

\section{K. Kalyanasundaram and M. Grätzel, Lausanne}

(EPFL Institute of Chemical Physics)

With the advent of lasers, there are hardly any areas in chemical physics that have not advanced considerably. Of particular interest is the ability to time-resolve molecular events in photochemistry and it is now possible to study the physical and chemical behaviour of light-induced transient species that last only a few nanoseconds. Laser applications to photochemistry are rather extensive, and we restrict ourselves here to the use of pulsed lasers in flash photolysis where short laser light pulses are used to initiate excited state processes. The events that follow are monitored both spectroscopically and kinetically on very short time scales - from a few picoseconds to several hundred nanoseconds.

The excited state species that are produced upon light (visible or UV) excitation are better understood in terms of the Jablonski diagram (Fig. 1). Consider the possibilities available for a "typical molecule" following absorption of a quantum of light of sufficient energy to place it in an excited vibrational level of a higher singlet excited state $\left(\mathrm{S}_{\mathrm{n}}\right)$ in the single manifold. The molecule will lose some of the excess energy rapidly in collisions - both by "vibrational relaxation" and "internal conversion" (on time scales $10^{-12}-10^{-11} \mathrm{~s}$ ) until it reaches the $v=0$ level of the lowest singlet excited state $S_{1}$. It may then return to the ground state $\left(\mathrm{S}_{0}\right)$ directly via either a radiative ("fluorescence") or a non-radiative process (time scales $10^{-9}-10^{-7} \mathrm{~s}$ ). Alternatively (or concurrently) the molecule in the excited state $\mathrm{S}$, can cross over to some higher vibrational levels of the triplet sublevels (usually $T_{1}$ ) by the process "intersystem crossing". From the triplet state it may eventually return to the ground state $\mathrm{S}_{0}$ via either a radiative ("phosphorescence") or a non-radiative process (on time scales $10^{-6}-10^{-3} \mathrm{~s}$ ). In the presence of other non-excited molecules (of the same kind or other), the molecule in the excited state $\mathrm{S}_{\text {, }}$ or $T_{1}$ can transfer the excited state energy or undergo chemical reactions such as electron-transfer and complex formation. By means of pulsed laser photolysis, it is possible to study all these processes accurately.

\section{Technique of Flash Photolysis}

In a typical flash photolysis set-up, a Q-switched laser, which can be a Nd or ruby or even a dye laser, delivers pulses of 5-20 ns and the events that follow the excitation of the sample are monitored by fast absorption kinetic spectroscopy. Here, the analysing beam from a pulsed $\mathrm{Xe}$ lamp is focused onto the sample (perpendicular or collinear to the direction of the laser beam) and, after transmission, is monochromatised and then refocused onto a photomultiplier of a few ns response time. In picosecond spectroscopy, the exciting pulses are used as analysing source by converting a part of the incident beam into a continuum, and passing through an optical delay line. Picosecond trains of pulses from a modelocked dye laser have also been used with time-correlated single photon counting, for time-resolved fluorescence studies.

\section{Excited State Characterization}

Detailed studies can be made of the singlet, triplet excited states (their absorption spectra $\mathrm{S}_{1} \rightarrow \mathrm{S}_{n}, \mathrm{~T}_{1} \rightarrow \mathrm{T}_{n}$, lifetimes and quantum yields of radiative processes such as fluorescence, phosphorescence) and their mutual cross-over processes such as inter-system crossing, internal conversion, vibrational relaxation and radiationless transfer. Laser photolysis has been applied extensively to small inorganic systems and large organic molecules such as condensed aromatic hydrocarbons and organic carbonyl compounds. As a result, it is now possible to construct an energy level diagram of the type shown in Fig. 1 with all the rate parameters known precisely for a wide variety of molecular systems. Fig. 2 presents, for example, the absorption spectrum $\left(T_{1} \rightarrow T_{n}\right)$ of the charge-transfer excited state of the transition metal-complex $\mathrm{Ru}(\mathrm{bpy})_{3}^{2+}$ in water, recorded at the end of the $20 \mathrm{~ns} 530 \mathrm{~nm} \mathrm{Nd}-3$ laser pulse excitation:

$$
\mathrm{Ru}(\mathrm{bpy})_{3}^{2+} \underset{\mathrm{hv}}{\rightarrow}\left[\mathrm{Ru}(\mathrm{bpy})_{3}^{2+}\right]^{*}
$$

The excited state which gives rise to this absorption spectrum has an emission lifetime of $650 \mathrm{~ns}$ in aqueous solution at room temperature. The phosphorescence emission can also be followed kinetically.

\section{Excited State Interactions}

In addition to the normal decay processes above, in the presence of other reactants (quenchers) the excited state species (singlets-triplets) can undergo a variety of reversible/irreversible chemical reactions. These can be broadly classified into three main categories.

(a) Quenching or more rapid deactivation of the excited state by interaction with ground state molecules of the same or another type:

${ }^{*} \mathrm{~S}+\mathrm{Q} \leftrightarrows \mathrm{S}+\mathrm{Q}^{*}$ (energy-transfer)

${ }^{*} \mathrm{~S}+\mathrm{Q} \leftrightarrows \mathrm{S}^{+}+\mathrm{Q}^{-}$(electron-transfer of excitation energy)

The de-activation can lead to a transfer of excitation energy to the quencher or transfer of electrons (oxidation or reduction). Verification of the various energy transfer mechanisms $(\mathrm{S} \rightarrow \mathrm{S}, \mathrm{S} \rightarrow \mathrm{T}$, $T \rightarrow T$, Forster-type, Dexter-type: dipoleinduced or resonance-induced) as well as the distinction as to whether or not a given quenching process is an electron or energytransfer requires direct monitoring of the possible transient species and this is easily done by the flash photolysis technique with pulsed lasers.

Fig. 3 presents typical oscilloscope traces showing kinetic behaviour of the transients during the following photoredox reaction, (initiated with a $20 \mathrm{~ns} 530 \mathrm{~nm} \mathrm{Nd}$ laser pulse) :

${ }^{3}\left(\mathrm{Ru}(\mathrm{bpy})_{3}^{2+}\right)^{*}+\mathrm{MV}^{2+} \underset{\mathrm{k}_{-1}}{\stackrel{\mathrm{k}_{\mathrm{q}}}{\leftrightarrows} \mathrm{Ru}(\mathrm{bpy})_{3}^{3+}}+\mathrm{MV}+$

Fig. 1 - Energy level diagram showing inter-relationship of various excited states that are formed upon light excitation of a molecule ("Jablonski Diagram").

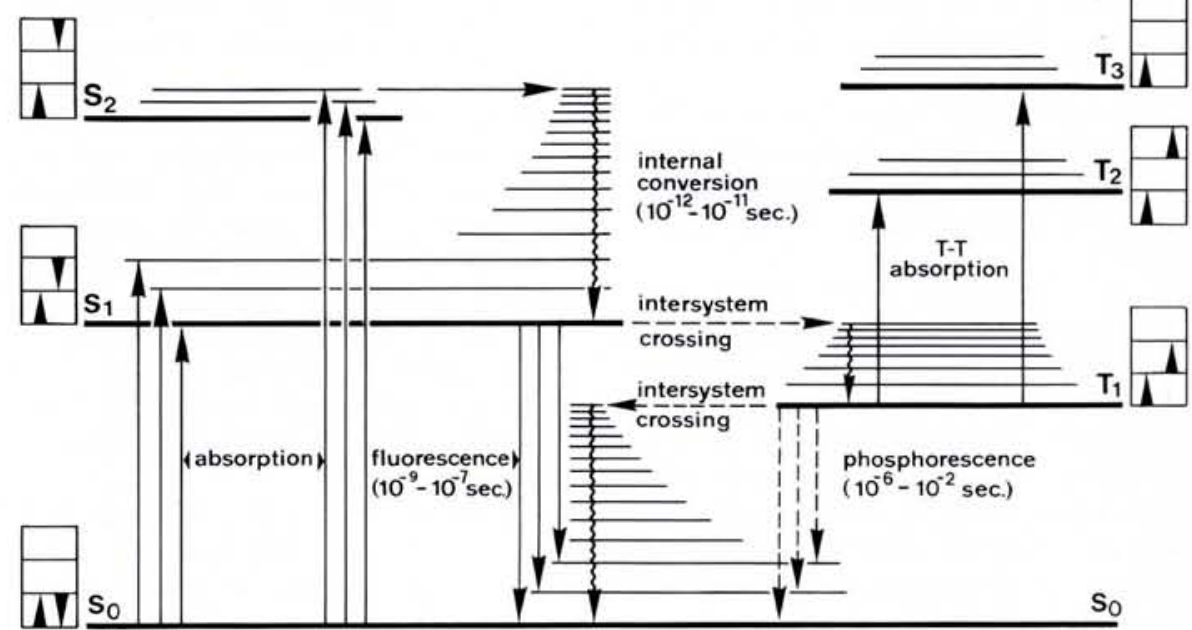




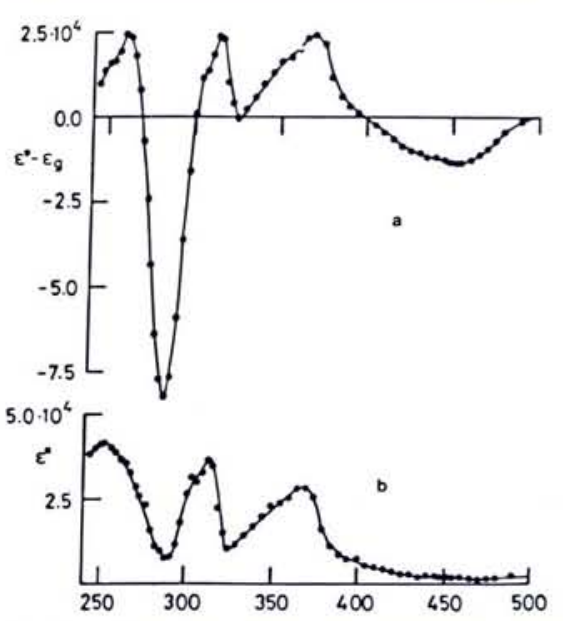

Fig. 2 - Transient absorption spectrum of the luminescent charge transfer excited state of the metal complex, Tris-bipyridyl ruthenium (II), $R u(b p y)_{3}^{2+}$ in water at room temperature recorded at the end of laser pulse. (a) difference $a b-$ sorption spectrum, (b) absorption spectrum after correction for the ground state absorption.

The forward reaction can be followed either by monitoring the enhanced decay of luminescence of ${ }^{3} \mathrm{Ru}(\mathrm{bpy})_{3}^{2+}$ at $\lambda=615$ $\mathrm{nm}$ (trace (a)) or by the rate of formation of the products, e.g. MV ${ }^{+}$, optically monitoring its growth at its absorption maximum in the visible (at $\lambda=600 \mathrm{~nm}$ ), (trace (b)). The redox products of the photoreaction $\mathrm{Ru}(\mathrm{bpy})_{3}^{3+}$ and $\mathrm{MV}$. recombine slowly in the dark and the rate constant for the back reaction measured by monitoring the decay of $\mathrm{MV}^{+}$absorptions at $\lambda=600 \mathrm{~nm}$ or of | Ru(bpy) $3_{3}^{3+}-\mathrm{Ru}(\mathrm{bpy})_{3}^{2+} \mid$ at $\lambda=457 \mathrm{~nm}$ (curve (c)). Kinetic analysis of the curves yields the rate constants for forward and reverse transfer as $4.0 \times 10^{8} \mathrm{M}^{-1} \mathrm{~s}^{-1}$ and $2.0 \mathrm{x}$ $10^{9} \mathrm{M}^{-1} \mathrm{~s}^{-1}$, respectively. The quantum yield of the excited state ${ }^{3} \mathrm{Ru}(\mathrm{bpy})_{3}^{2+}$ and of the redox products $\mathrm{MV} \pm$, Ru(bpy) $3_{3}^{+}$can be shown to be 1.00 and 0.30 respectively in water.

(b) Formation of the excited state associated dimers or complexes (excimers and exciplexes):

$\mathrm{S}+\mathrm{S} \rightarrow \mathrm{S}_{2}^{*} \rightarrow$ emission $\mathrm{S}_{2}$

(excimer)

${ }^{*} S+D \rightarrow(S D)^{*} \underset{\text { emission }}{\rightarrow} S+D$ (exciplexes)

To explain some experimental results, Th. Forster postulated the possible formation of these excimers and exciplexes in 1955. By quantitative studies of their absorption spectra, lifetimes and yields by pulsed laser techniques, especially due to Mataga and Ottolenghi, these associated excited species are now as well characterized as their parent species: singlets and triplets. Typical examples are excimers of hydrocarbons such as pyrene and exciplexes derived from aromatic hydrocarbon amines.

(c) Photoionization from the excited state by one, two, ... multiphoton processes:

$S^{*} \stackrel{\text { hy }}{\rightarrow} \mathrm{S}^{+}+\mathrm{e}^{-}$

The photoionization process is monitored by following the growth and decay of the cations and electrons by optical absorp- tion. An equivalent and elegant method in use is pulsed laser conductivity studies, where instead of absorptions, changes in the conductivity of the solutions upon laser light excitations are monitored on very fast scales. By this means, extensive information has been obtained on the mechanisms of mono, biphotonic processes as well as on the dynamical behaviour of electrons in liquids (mobility, trapping, etc.).

\section{Application to Photochemistry and Photobiology}

In addition to their routine use to monitor the kinetic behaviour of singlet and triplet excited states, the technique of laser flash photolysis has been exploited to assimilate extensive information on several areas of organic and inorganic photochemistry as well as in photobiology. Particular attention here can be paid to the recent advances in the chemistry of singlet oxygen, photoreactivity of carbonyl compounds, photochemistry of transition metal complexes, especially those with bipyridine or phenanthroline ligands, fluorescence probe analysis of aggregate systems such as micelles, vesicles, polymers and polyelectrolytes, primary processes of photosynthesis and photochemistry of visual pigments such as retinals and rhodopsin. Space limitations preclude any detailed discussions of these.

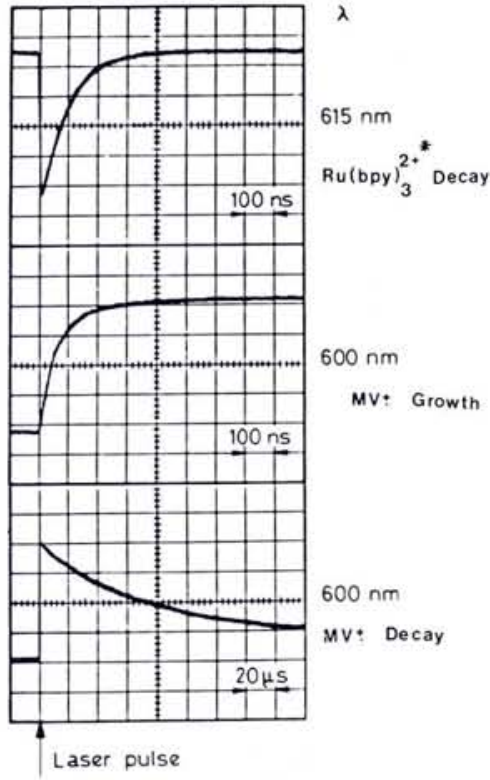

Fig. 3 - Oscilloscope traces showing the kinetic behaviour of the transients following electrontransfer quenching of $3\left[R u(b p y)_{3}^{2+}\right]^{*}$ excited state by methylviologen (MV $\left.{ }^{2+}\right)$.

a) decay of ${ }^{3}\left[R u(b p y)_{3}^{2+}\right]^{*}$ emission monitored at $\lambda=615 \mathrm{~nm}$.

b) growth of $M V+$ radical, monitored at $\lambda=600 \mathrm{~nm}$.

c) decay of $M V+$ radical at longer time scale (back transfer).

\title{
Isotope Separation
}

\section{F.S. Becker and K.L. Kompa, Garching}

\author{
(Max-Planck Institute for Quantum Optics)
}

Isotope separation is today one of the most actively pursued areas of laser application. The advent of laser excitation sources at many different vvavelengths, with high spectral purity and high energy density has revolutionized concepts of photophysical or photochemical isotope separation, which were, in principle, known long ago but could never be put into practice because of the limited source quality of conventional lamps. Many isotopes have been separated successfully to date in laboratory experiments, but much still remains to be done to extend the results to an industrial scale, where the process is optimized for economy.

There are many problems in science which can be tackled if high quality pure isotopes are available, while the enrichment of uranium in the isotope ${ }^{235} \mathrm{U}$ is of considerable importance to nuclear technology.

The most important differences between the isotopic constituents of an atomic or molecular mixture are spectral, which opens the possibility of directing the energy needed for separation, to the isotope that is to be extracted. In any isotope separation scheme the following requirements must be satisfied:

1) The absorption spectrum must exhibit an isotope shift, sufficiently large in relation to the spectral line or band contours.

2) A laser light source or combination of sources must be available with appropriate wavelengths, monochromaticity and intensity.

3) The selective excitation of the desired species must lead to an irreversible separation process via a physical/chemical follow-up procedure.

4) The selectivity of the entire sequence of excitation and separation steps must not significantly be impaired by inter-isotope energy transfer and scrambling.

Spectral isotope shifts are found in both atomic and molecular spectra. We shall concentrate in the following on the latter and, in particular, on the use of infrared laser induced multiphoton absorption to dissociate the desired isotopic compound.

\section{Basic Spectroscopic Prerequesites}

In 1975 it was discovered by several groups that the incidence of high power infrared laser radiation on molecular gases, 